\title{
Patterns of Polypeptide Synthesis in Non-fruiting Monokaryons and a Fruiting Dikaryon of Schizophyllum commune
}

\author{
By O. M. H. DE VRIES* AND J. G. H. WESSELS \\ Department of Developmental Plant Biology, Biological Centre, University of Groningen, Haren, \\ The Netherlands
}

(Received 6 May 1983)

The patterns of polypeptides synthesized by two co-isogenic monokaryons and the derived dikaryon of the basidiomycete Schizophyllum commune, growing in surface culture, were compared by two-dimensional PAGE after labelling with $\left[{ }^{35} \mathrm{~S}\right]$ sulphate. Synthesis of most of the polypeptides ( 400 analysed) was common but at day 3 some 30 polypeptides were synthesized in the dikaryon which were not seen in the monokaryons. At this time the dikaryon had formed hyphal aggregates. Ten additional novel polypeptides were being synthesized in the primordia arising from these aggregates at day 4.

In surface culture, monokaryons and dikaryon excreted 4 to $8 \%$ of the labelled proteins (over 80 different polypeptides) into the medium compared with only 1 to $2 \%$ when cultivation was done in liquid shaken cultures. The polypeptides specifically secreted by the dikaryon at the aggregation stage consisted of seven small polypeptides with molecular weights ranging from 10 to $14 \times 10^{3}$, and two polypeptides with molecular weights $18 \times 10^{3}$ and $26 \times 10^{3}$, respectively. Such differences in excreted proteins between monokaryons and dikaryon were not observed when the mycelia were grown in liquid shaken cultures, indicating a relationship with fruit-body formation.

A comparison of labelled proteins in isolated hyphal walls revealed six polypeptides specifically associated with the walls of monokaryons and dikaryon irrespective of the cultivation method. Among the polypeptides newly appearing in the dikaryon at aggregation two were associated with the walls and co-migrated with the polypeptides of molecular weights $18 \times 10^{3}$ and $26 \times 10^{3}$ excreted into the medium at this stage of development.

\section{INTRODUCTION}

Previous studies in our laboratory have indicated that the cellular differentiation between monokaryon and dikaryon in the basidiomycete Schizophyllum commune, when grown in shaking culture, is accompanied by small changes in the patterns of protein synthesis as detected on two-dimensional gels (De Vries et al., 1980a). Under the same conditions the corresponding mRNA populations were almost identical as shown by their in vitro translation products (Hoge et al., 1982a). Moreover, the entire RNA complexities comprising about 10000 different average-sized RNA sequences, were found to be similar in the two mycelial types (Zantinge $e t$ al., 1979, 1981; Hoge et al., 1982a). However, some 20 specific translatable RNAs belonging to the class of abundant mRNAs were found to appear in the dikaryon at the time of primordia formation (Hoge et al., 1982b).

We therefore examined the patterns of protein synthesis in vivo of monokaryons and dikaryon at the time of primordial development in the dikaryon. Rather extensive differences were found in the synthesis of extracellular proteins, some of which appear firmly associated with cell walls. 


\section{METHODS}

Organism. The co-isogenic monokaryons 4-39 (A4lB4I) and 4-40 (A43B43) (CBS 341.81 and CBS 340.81, respectively) and the dikaryon 4-39/4-40 obtained by mating these monokaryons were used (Zantinge et al., 1979).

Culture methods. Surface cultures were grown in small Petri dishes (diam. $5.3 \mathrm{~cm}$ ) containing $15 \mathrm{ml}$ minimal medium (Dons et al., 1979) supplemented with $1.5 \%(\mathrm{w} / \mathrm{v})$ agar. The plates were covered with permeable cellophane (300P, British Cellophane Ltd. Bridgewater, UK). To analyse total proteins including excreted proteins, the cellophane was overlaid with $2 \mathrm{ml}$ of the same agar medium. For extracellular and wall-associated proteins the mycelium was grown directly on the cellophane. Plates were inoculated by spreading $0.5 \mathrm{ml}$ of a suspension of macerated mycelium on the surface of the cellophane or the agar. Cultivation was at $24^{\circ} \mathrm{C}$ under continuous light. After $3 \mathrm{~d}$ when about half of the glucose in the medium had disappeared, the cellophane sheets bearing the mycelial mats were transferred to fresh agar plates to ensure unlimited nutrition. Liquid cultures were grown in minimal medium on a reciprocal shaker (250 r.p.m.) as described by Zantinge et al. (1979).

Labelling and harvesting. Surface cultures were labelled by transferring the mycelial mats without the cellophane to small Petri dishes containing $1 \mathrm{ml}$ minimal medium in which $\mathrm{MgCl}_{2}$ was substituted for $\mathrm{MgSO}_{4}$ and to which was added $\left[{ }^{35} \mathrm{~S}\right]$ sulphate $\left(3.7 \times 10^{7} \mathrm{~Bq}, 150 \mathrm{GBq}\right.$ mmol ${ }^{-1}$, Amersham) or $\left[{ }^{35}\right.$ S methionine $\left(1.5 \times 10^{6} \mathrm{~Bq}\right.$, $18.5 \mathrm{GBq} \mathrm{mmol}{ }^{-1}$, New England Nuclear). Labelling was mostly done with $\left[{ }^{35} \mathrm{~S}\right]$ sulphate because preliminary experiments showed that labelling with $\left[{ }^{35}\right.$ S $]$ methionine resulted in rapid labelling of the vegetative mycelia but with little translocation of the label into the developing primordia. The label was applied for 4 to $5 \mathrm{~h}$ at $24^{\circ} \mathrm{C}$. For the analysis of total proteins in primordia and in the mat carrying them (including extracellular proteins), the whole mat with adhering agar was first frozen in liquid nitrogen, placed on a copper plate kept at $-10^{\circ} \mathrm{C}$, and the primordia were scraped off with a chisel and stored separately at $-70^{\circ} \mathrm{C}$. If wall-associated proteins were to be analysed, the mycelium was grown directly on cellophane and the labelled mycelial mats were quickly washed with five $100 \mathrm{ml}$ portions of minimal medium, frozen in liquid nitrogen, and stored at $-70^{\circ} \mathrm{C}$. The medium used for labelling was analysed for extracellular proteins excreted into the medium.

Preparation of extracts. For total proteins, frozen hyphal material was fragmented in an X-press at $-25^{\circ} \mathrm{C}$ (LKB) and immediately extracted in 2 vol. SDS-sample buffer [final concn $2 \%(w / v)$ SDS, $5 \%$ (v/v) 2mercaptoethanol, $0.05 \mathrm{M}$-Tris $/ \mathrm{HCl}$ buffer $\mathrm{pH} 6.8,10 \%(\mathrm{v} / \mathrm{v})$ glycerol] at $100{ }^{\circ} \mathrm{C}$ for $5 \mathrm{~min}$. The extracts were cleared by centrifugation (Eppendorf microfuge, $3 \mathrm{~min}$ ).

To detect proteins in hyphal walls, frozen X-press fragmented hyphal material $(0.2 \mathrm{~g}$ wet wt) was quickly thawed and suspended on a Vortex mixer in $5 \mathrm{ml} 0.1 \mathrm{M}$-Tris $/ \mathrm{HCl}, \mathrm{pH} 7 \cdot 6$, containing $1 \mathrm{~mm}$-phenylmethanesulphonyl fluoride to inhibit proteolytic degradation (cf. Pringle, 1975). After centrifugation $(1000 \mathrm{~g}, 5 \mathrm{~min})$ the pellet was washed 12 times with $5 \mathrm{ml}$ cold $0 \cdot 1 \mathrm{M}$-Tris/ $\mathrm{HCl}, \mathrm{pH} 7 \cdot 6$. Wall-associated proteins were then extracted with SDS-sample buffer $(0.5 \mathrm{ml})$ at $100^{\circ} \mathrm{C}$ for $5 \mathrm{~min}$.

To monitor excreted proteins the medium to which the label had been added was also adjusted to the same concentration as SDS-sample buffer and heated at $100^{\circ} \mathrm{C}$ for $5 \mathrm{~min}$. To measure hot-TCA insoluble radioactive material, samples were spotted on Whatman $3 \mathrm{MM}$ filters pre-treated with $0.1 \mathrm{M}-\mathrm{MgSO}_{4}, 0.1 \%(\mathrm{w} / \mathrm{v})$ methionine and $0.1 \%(\mathrm{w} / \mathrm{v})$ cysteine. In the case of extracellular liquids, the filters were also preloaded with $50 \mu \mathrm{g}$ bovine serum albumin as a carrier. The filters were dried and treated with boiling $10 \%(\mathrm{w} / \mathrm{v}) \mathrm{TCA}$ containing $0 \cdot 1 \%(\mathrm{w} / \mathrm{v})$ methionine and $0 \cdot 1 \%(\mathrm{w} / \mathrm{v})$ cysteine, washed with the same solution but $5 \%(\mathrm{w} / \mathrm{v})$ in TCA, rinsed with $96 \%(\mathrm{v} / \mathrm{v})$ ethanol and counted by liquid scintillation.

Two-dimensional gel electrophoresis. This was carried out as described by O'Farrell (1975) after adjusting the samples to $0.4 \%(\mathrm{w} / \mathrm{v})$ SDS, $2 \%(\mathrm{v} / \mathrm{v})$ glycerol, $9 \cdot 5 \mathrm{M}$-urea, $5 \%(\mathrm{v} / \mathrm{v})$ Nonidet $\mathrm{P} 40,5 \%(\mathrm{v} / \mathrm{v}) 2$-mercaptoethanol, $1.6 \%(\mathrm{v} / \mathrm{v})$ ampholines (LKB) $\mathrm{pH} \mathrm{5-7.} \mathrm{and} 0.4 \%(\mathrm{v} / \mathrm{v})$ ampholines $\mathrm{pH} 3.5-10$. In this way proteins in hot-SDS extracts can also be separated by iso-electrofocusing in the first dimension as described (O'Farrell, 1975). The first dimension resolved polypeptides with iso-electric points ranging from $\mathrm{pH} 4.5$ to 6.5 . For the second dimension $10 \%(w / v)$ polyacrylamide gels in the presence of SDS were chosen to fractionate and analyse polypeptides with molecular weights ranging from 20 to $130 \times 10^{3}: 12.5^{\circ}(\mathrm{w} / \mathrm{v})$ gels were used to resolve polypeptides with molecular weights ranging from 10 to $30 \times 10^{3}$. Samples to be compared contained equal amounts of radioactivity in hot-TCA precipitable material. Electropherograms were fluorographed at $-70^{\circ} \mathrm{C}$ using preflashed film (Laskey \& Mills, 1975). Patterns of accumulated proteins were visualized by using the sensitive silver staining procedure essentially as described by Wray et al. (1981). The latter staining procedure was also applied to gels which had been previously prepared for fluorography. In these cases the gel was released from the paper by soaking in $30 \%(\mathrm{v} / \mathrm{v})$ ethanol. PPO was dissolved during incubation overnight in $100 \mathrm{ml}$ DMSO, followed by $3 \mathrm{~h}$ in $100 \mathrm{ml}$ fresh DMSO. The gel was then transferred to distilled water, any adhering paper fibrils being carefully removed, and silver stained

Peptide mapping. Proteins of interest were cut from dried PPO-impregnated gels and peptide mapping using Staphylococcus V8 protease (Miles, Stoke Poges, UK) was carried out according to the procedure described by Cleveland et al. (1977) for digestion of proteins in gel slices in the presence of SDS. Digestions were done at 24 " $\mathrm{C}$ with 0.01 and $0.1 \mu \mathrm{g} \mathrm{V8}$ protease in the presence of $15 \mu \mathrm{g}$ myoglobin (type 1 , Sigma) per slot. Fragments were separated on $17 \cdot 5^{\circ}(\mathrm{w} / \mathrm{v})$ polyacrylamide gels and detected by fluorography. 
Table 1. Classification of differences between two-dimensional patterns of total polypeptides* synthesized during a $5 \mathrm{~h}$ pulse with $\left[{ }^{35} S\right]$ sulphate in co-isogenic monokaryons and derived dikaryon of $S$. commune after $4 d$ growth in surface cultures

\begin{tabular}{cccc} 
Monokaryons & \multicolumn{2}{c}{ Dikaryon } & $\begin{array}{c}\text { Number of } \\
\text { differences }\end{array}$ \\
\cline { 2 - 4 } $\begin{array}{c}\text { Culture minus } \\
\text { primordia }\end{array}$ & $\begin{array}{c}\text { Isolated } \\
\text { primordia }\end{array}$ & 22 \\
- & + & + & 15 \\
- & - & + & 8 \\
+ & - & - & 3 \\
+ & + & - & 0 \\
- & + & - & 0
\end{tabular}

* The total number of spots analyzed was 400 ; fractionation range: iso-electric points, $\mathrm{pH} 4.5$ to 6.5 ; molecular weights, $10 \times 10^{3}$ to $130 \times 10^{3}$ (cf. Figs 1 and 2 ). With respect to the classification each row indicates a certain category of polypeptides. For instance, the first row indicates that 22 polypeptides can be found in both the dikaryon minus the primordia and in the isolated primordia but not in the monokaryons, while the last row but one indicates that no polypeptides were present in the culture minus primordia that were absent from both the isolated primordia and the monokaryons.

\section{RESULTS}

\section{Comparison of patterns of protein synthesis of monokaryons and differentiating dikaryon}

Four day surface cultures of the co-isogenic monokaryons and the derived dikaryon were labelled with $\left.{ }^{35} S\right]$ sulphate for $5 \mathrm{~h}$. The numerous primordia (approximately $50 \mathrm{~cm}^{-2}$ ) present in the dikaryon were separated from the mycelial mat. Extracts were prepared from the monokaryons and from the primordia and the mycelial mat of the dikaryon. The specific activities of these extracts were approximately the same [about 600 c.p.m. ( $\mu$ g protein) ${ }^{-1}$ ]. These extracts were fractionated by two-dimensional gel electrophoresis using 10 and $12.5 \%$ polyacrylamide-SDS gels for the second dimension, and the fluorograms analysed. Within the fractionation range (iso-electric points $\mathrm{pH} 4.5$ to 6.5 ; molecular weights 10 to $130 \times 10^{3}$ ) the analysis encompassed 400 spots (Fig. 1 and Fig. $2 a b$ ). The overall qualitative differences are summarized in Table 1. The presence of 37 unique polypeptides was conspicuous in the dikaryon at the time of fruit-body formation; 22 of these were found both in the extracts of the primordia and the mat [arrows pointing down and arrowheads labelled (CW1) and (CW2) in Fig. $1 b, c$ and $2 b$ ], whereas 15 polypeptides were exclusively present in the primordia (arrows pointing up in Fig. 1c). Only eight polypeptides were found to be specifically synthesized in the monokaryons (arrowed in Fig. $1 a$ ).

We also analysed the occurrence of the 37 dikaryon-specific polypeptides in $3 \mathrm{~d}$ old cultures of the dikaryon after $5 \mathrm{~h}$ pulse labelling with $\left.{ }^{35} \mathrm{~S}\right]$ sulphate (data not shown). At this stage the mycelium showed the presence of numerous aggregates, but cup-shaped primordia were not yet formed. Since the aggregates could not be separated from the mat, extracts of total cultures were analysed. It appears that all but 10 of the 37 dikaryon-specific polypeptides present at day 4 could be detected. Eight of these missing polypeptides belonged to the class of proteins of which the synthesis at day 4 was only detected in the primordia.

\section{Polypeptides excreted into the medium}

In the previous analyses the vegetative mycelium was analysed together with the thin adhering agar layer into which this mycelium had penetrated. Thus, differences in polypeptide synthesis as observed between monokaryons and dikaryon possibly included polypeptides excreted into the medium. Indeed, the liquid medium used for labelling mycelium directly grown on cellophane contained 4 to $8 \%$ of the total radioactivity incorporated into hot TCAprecipitable material.

Two-dimensional analysis of the extracellular material revealed a rather complex pattern of more than 80 different polypeptides. Monokaryon and dikaryon shared at least 65 polypeptides, the majority with iso-electric point between pH 4.5 and 5.5 . However, marked qualitative and 
quantitative differences were noticed, especially with regard to relatively small proteins with molecular weight between $10 \times 10^{3}$ and $15 \times 10^{3}$, which were only synthesized by the fruiting dikaryon (Fig. $2 c, d$ ). Seven polypeptides (arrowed in Fig. $2 d$ ) were invariably found to be excreted only by the dikaryotic mycelium (five independent labelling experiments). In addition two polypeptides of molecular weight $18 \times 10^{3}$ and $26 \times 10^{3}$ were found in much larger amounts in the medium of the dikaryon than in that of the monokaryons [labelled (CW1) and (CW2) in Fig. 2d]. As shown in Fig. 2(b) all these polypeptides can also be traced in patterns of total protein which includes extracellular proteins.

To see whether differences also arise in the absence of fruit-body development, we analysed the polypeptides excreted by monokaryons and dikaryon grown in liquid shaken cultures for $2 \mathrm{~d}$. Under these conditions the percentage of proteins excreted was found to be smaller than in surface cultures ( 1 to $2 \%$ of the total hot TCA-precipitable material) but the overall complexity of the two-dimensional patterns of the extracellular polypeptides was similar. However, none of the nine extracellular polypeptides specific for $4 \mathrm{~d}$ old surface cultures of the dikaryon were found to be present. In fact, the patterns of monokaryons and dikaryon were almost identical.

The nine extracellular polypeptides specific for the $4 \mathrm{~d}$ old dikaryon bearing primordia were found to be already excreted at day 3 during the aggregation stage. Therefore (some of) these polypeptides might be related to early stages of primordia formation, e.g. hyphal aggregation.

\section{Polypeptides associated with hyphal walls}

Extracellular proteins involved in cell-cell interactions are likely to be associated with hyphal walls. Therefore we isolated hyphal walls from $4 \mathrm{~d}$ old surface cultures of monokaryons and dikaryon labelled with $\left.{ }^{35} \mathrm{~S}\right]$ sulphate. After 12 washings with buffer the walls contained 10 to $15 \%$ of the total TCA-precipitable radioactivity. Washed walls from monokaryons and dikaryon were extracted with hot SDS-sample buffer and the extracts were two-dimensionally fractionated. Spot by spot comparison of these patterns with those of the first supernatant after

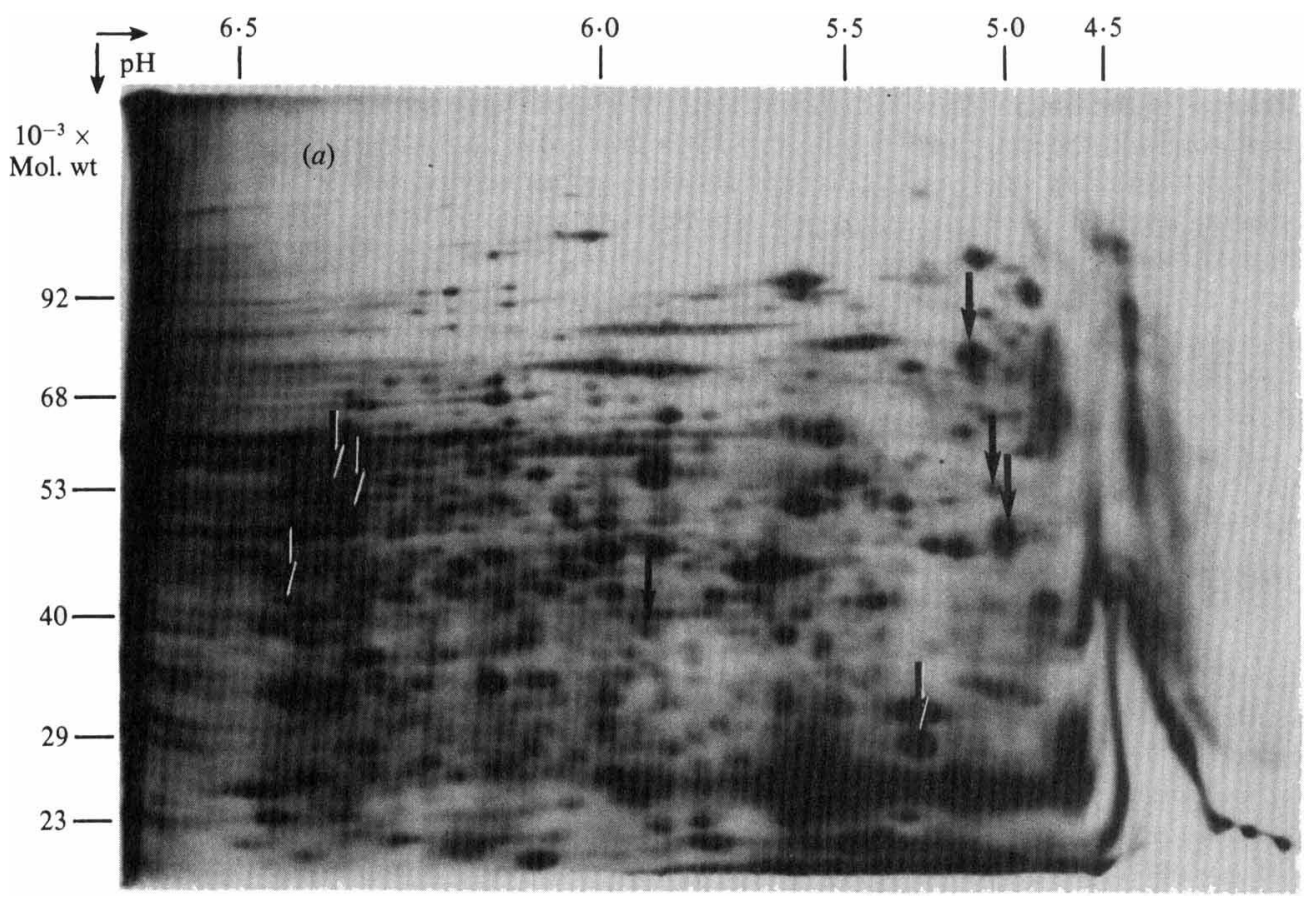



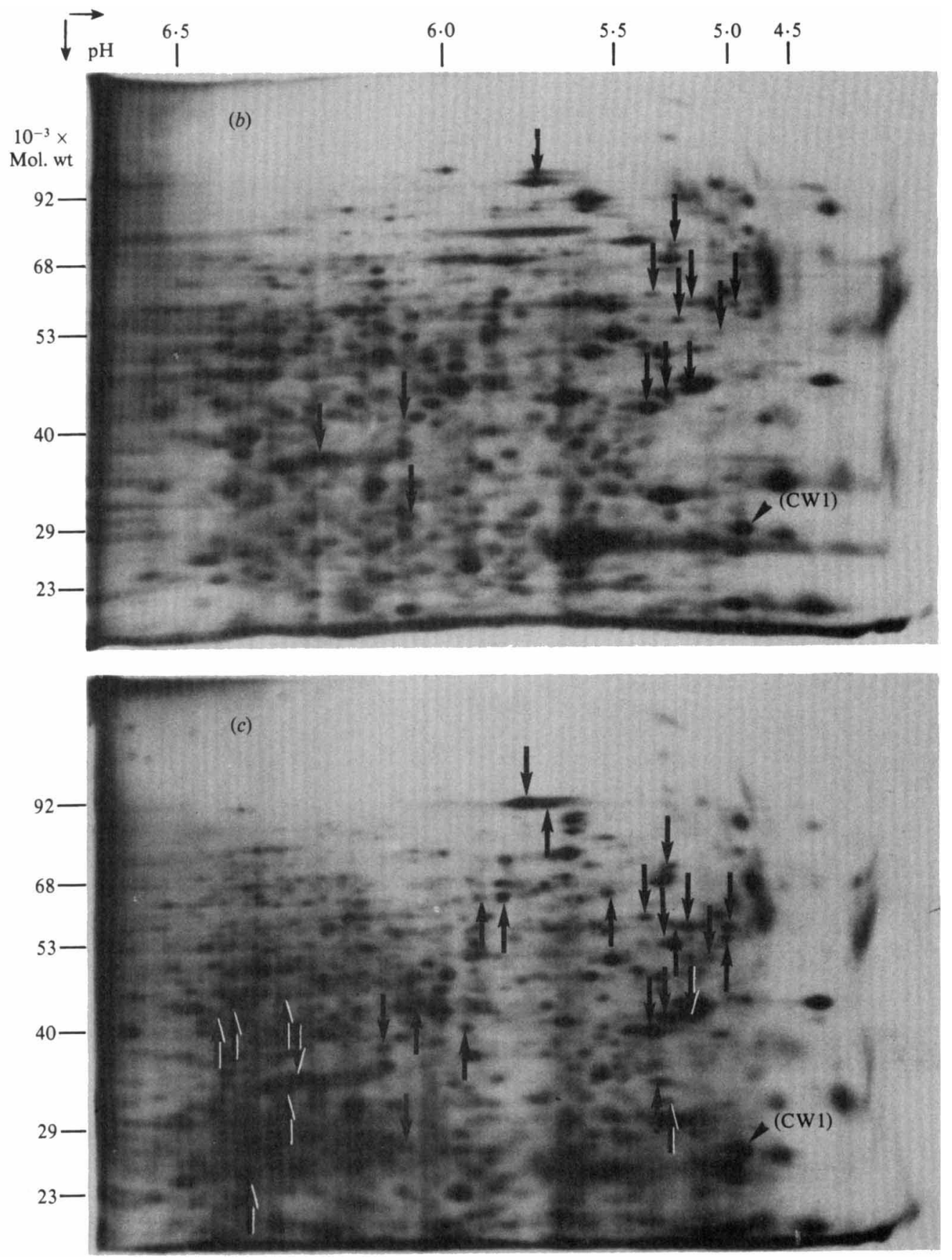

Fig. 1. Two-dimensional separations of total polypeptides synthesized by $4 \mathrm{~d}$ old surface cultures of Schizophyllum commune during a $5 \mathrm{~h}$ pulse with $\left[{ }^{35}\right.$ S $]$ sulphate. Extracts from : (a) vegetative co-isogenic monokaryons (mixed in equal amounts before separation); (b) dikaryotic mat (mycelium plus agarmedium) after removal of primordia ; $(c)$ primordia. For the second dimension $10 \%$ polyacrylamide gels were used. Monokaryon-specific polypeptides are arrowed in $(a)$, dikaryon-specific polypeptides occurring in both primordia and the mat are indicated in $(b)$ and $(c)$ with arrows pointing down, and primordia-specific polypeptides are indicated in $(c)$ with arrows pointing up. 

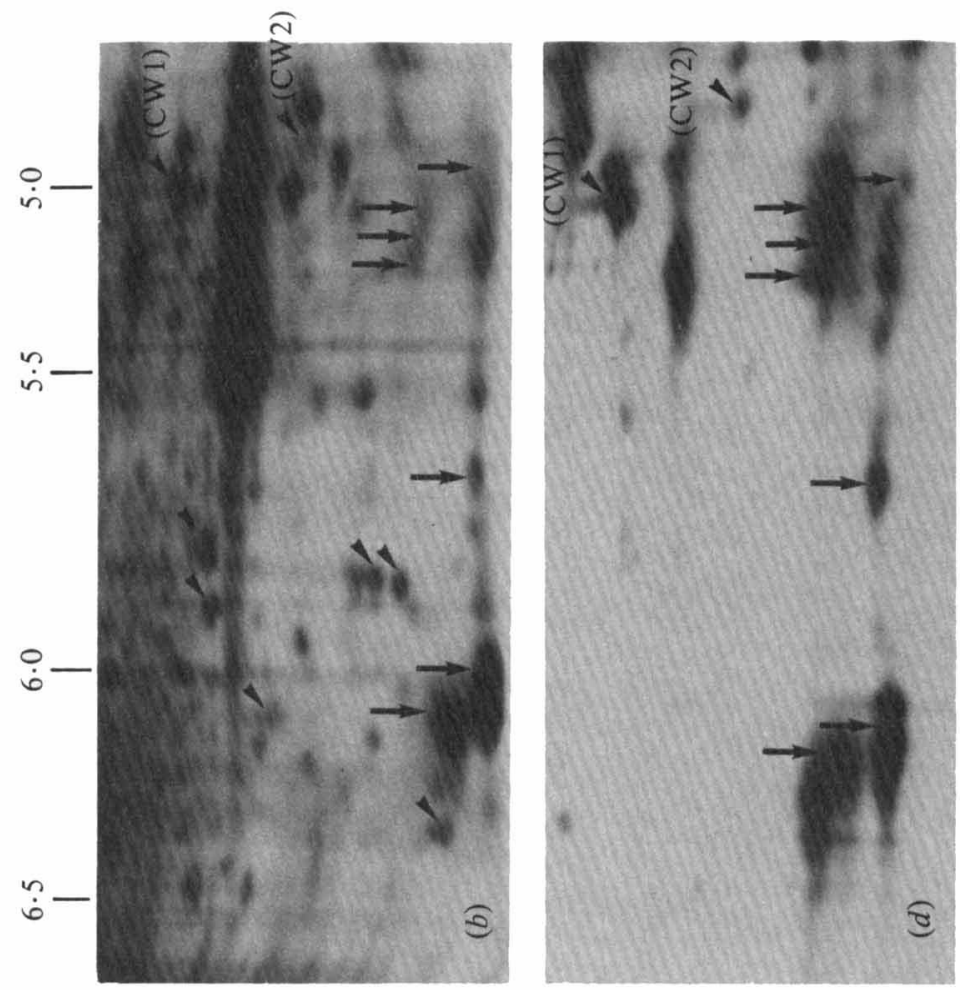

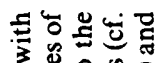

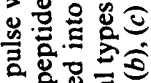

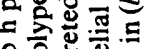

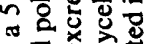
की 월 获 둔휴을 8. 3 눙형 을

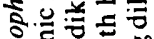

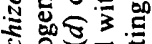
फ. . . . 웅 $\mathrm{g}$

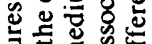

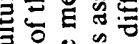
论蛅 过

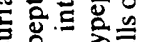
की 궁

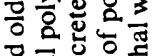

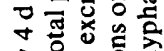

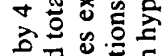
可语 证
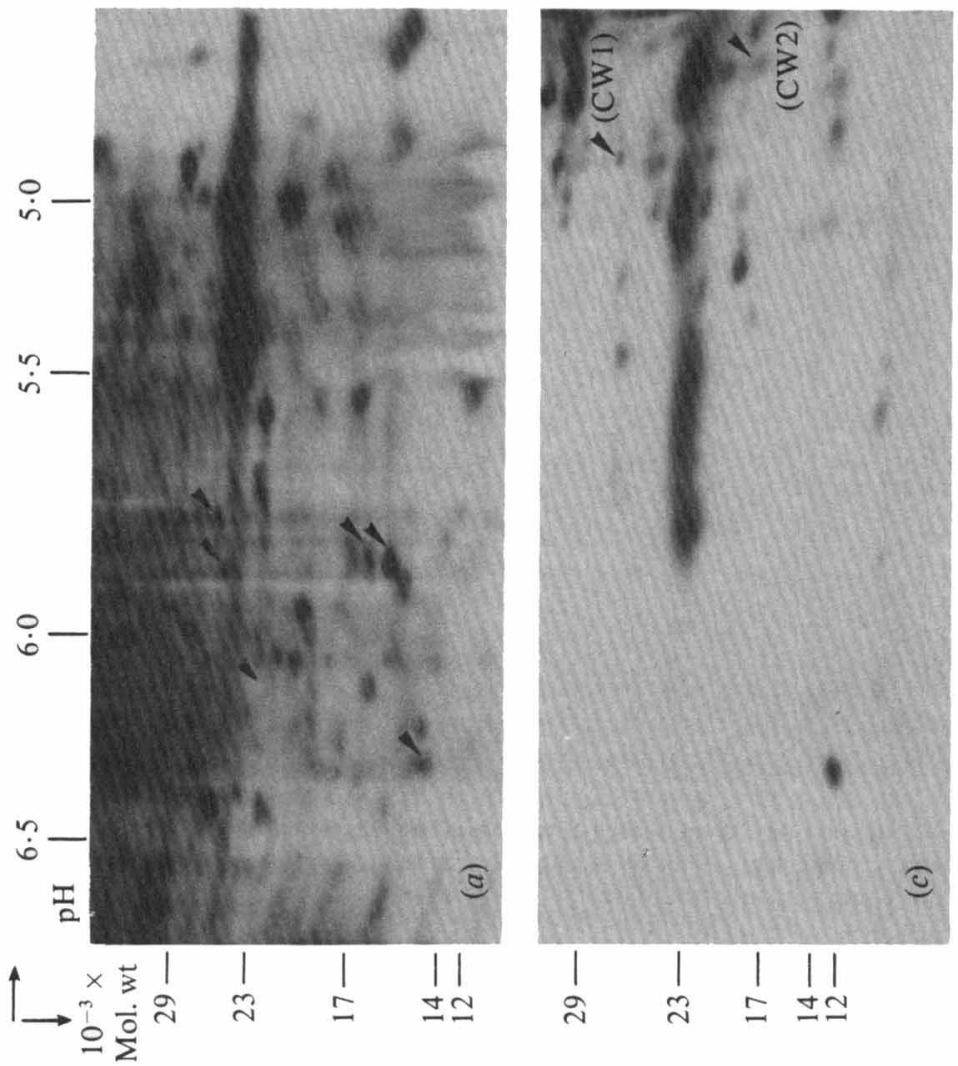
可产要 ลें

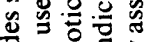
일.

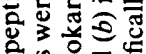

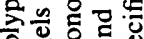
\&.

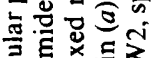

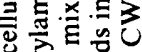
过苋 × 혀을 สํํ워

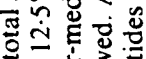
的

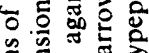
纯 结言范 형 둥르응

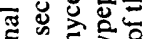

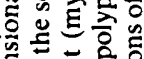
क 토 통 을

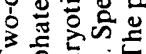

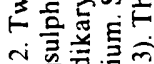

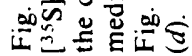



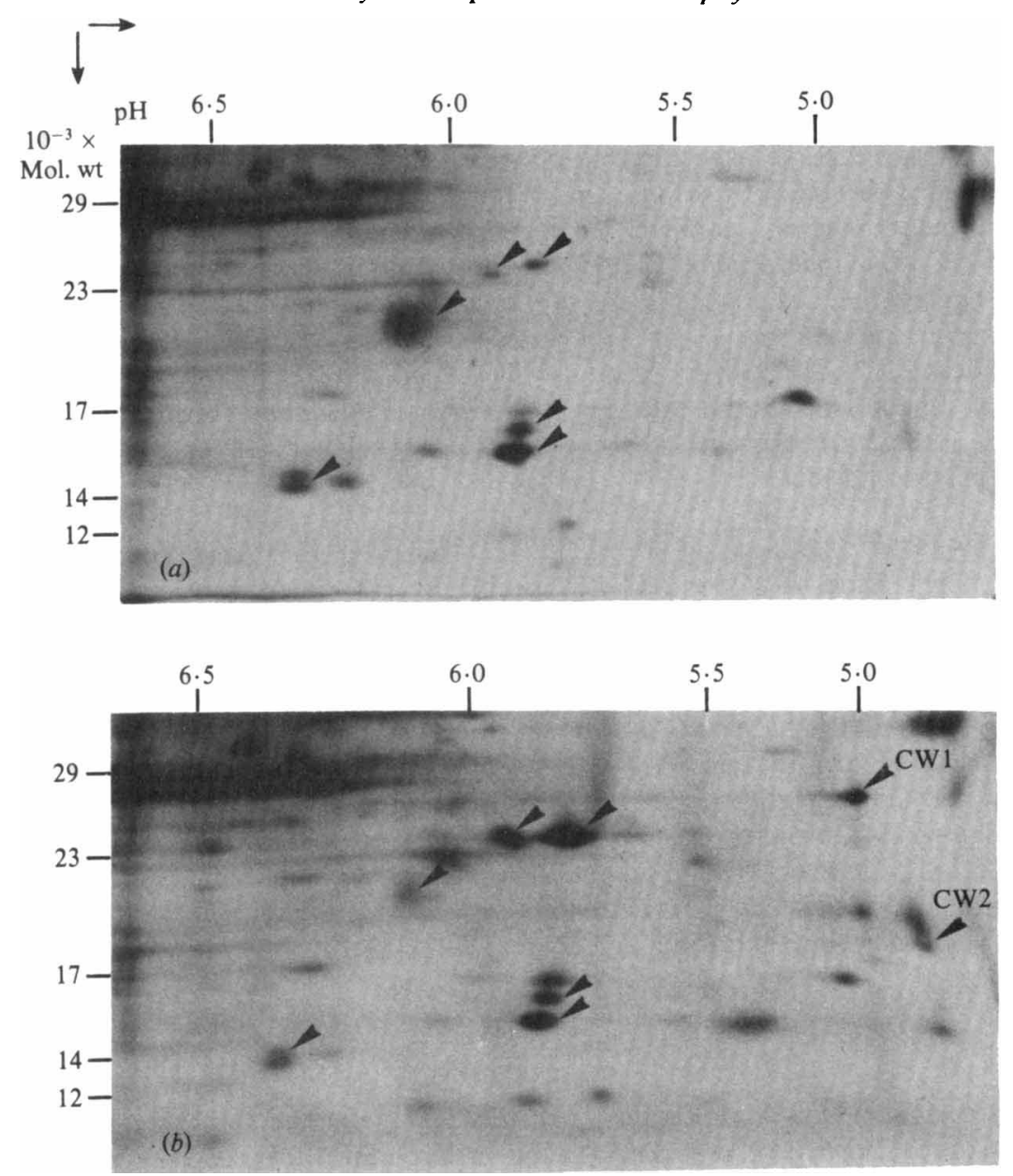

Fig. 3. Two-dimensional separations of polypeptides extracted from hyphal walls of $4 \mathrm{~d}$ old surface cultures of Schizophyllum commune after a $5 \mathrm{~h}$ pulse with $\left[{ }^{35} \mathrm{~S}\right] \mathrm{sulphate}$. The hyphal walls were isolated and extracted as described in Methods. (a) Mixed extracts of walls of the two co-isogenic monokaryons: (b) extracts of walls of the differentiating dikaryon. The six polypeptides specifically associated with hyphal walls of both mycelial types (i.e. not present in the cytoplasmic fraction) are indicated with arrowheads. The two polypeptides specifically associated with dikaryotic hyphal walls are indicated with arrowheads labelled CW1 and $\mathrm{CW} 2$.

breaking the hyphae (cytoplasmic fraction, results not shown) revealed six polypeptides specifically associated with the walls of both mycelial types and not present in the cytoplasmic fraction (Fig. 3, arrowheads). However, two additional polypeptides (Fig. 3b, arrowheads labelled $\mathrm{CW} 1$ and $\mathrm{CW} 2$ ) with molecular weights $26 \times 10^{3}$ and $18 \times 10^{3}$, respectively, were found in walls of the differentiating dikaryon. Most of these wall polypeptides can also be traced in the patterns of total proteins (arrowheads in Fig. $2 a, b$ ).

Further purification of the walls (further washings with sonification, treatments with $0.5 \%$ Nonidet P40 and/or DNAase 1) did result in the loss of radioactive material from the walls but with little further enrichment of the wall-specific polypeptides.

By using the sensitive silver-stain procedure (Wray et al., 1981) many of the wall proteins detected by fluorography became visible including CWI and CW2. No additional differences were found between these patterns, which represent the accumulated polypeptides in the hyphal walls of monokaryons and differentiating dikaryon. 
Walls isolated from the monokaryons and the dikaryon grown in liquid culture contain the same six polypeptides shared by the walls of monokaryons and dikaryon grown in surface culture. However, the two wall polypeptides $\mathrm{CW} 1$ and $\mathrm{CW} 2$ were conspicuously absent. On the other hand, the two dikaryon-specific wall polypeptides were found in surface cultures of the dikaryon grown in sealed Petri dishes. Under these conditions cup-shaped primordia do not form but instead numerous compact aggregates develop, heavily overgrown with aerial mycelium (Volz \& Niederpruem, 1970). Therefore these two wall polypeptides might be involved in aggregation rather than in any of the further morphogenetic processes operating in fruit-body construction.

Polypeptides CW1 and CW2 could also be labelled with $\left[{ }^{35}\right.$ S $]$ methionine instead of $\left.{ }^{[35} \mathrm{S}\right]$ sulphate although the relative intensity of $\mathrm{CW} 2$ was somewhat reduced. This indicates that the labelling of these proteins with $\left[{ }^{35} \mathrm{~S}\right]$ sulphate was not solely due to sulphate-substitution of any bound polysaccharides.

\section{Correspondence of $\mathrm{CW1}$ and $\mathrm{CW} 2$ with polypeptides excreted into the medium}

The two polypeptides $\mathrm{CW} 1$ and $\mathrm{CW}$, specifically associated with hyphal walls of the differentiating dikaryon (Fig. 3), co-migrated with polypeptides excreted by the differentiating dikaryon into the medium (arrowheads in Fig. $2 d$ ). Corresponding spots could also be recognized in fluorograms of total proteins of the differentiating dikaryon [arrowheads labelled (CW1) and (CW2) in Figs $1 b, c$ and $2 b$ ]. An indication that CW1 and CW2 were identified with the co-migrating polypeptides excreted into the medium by the differentiating dikaryon was obtained by analysing a peptide digest according to the procedure described by Cleveland $e t$ al. (1977) for digestion of proteins in gel slices in the presence of SDS using Staphylococcus V8 protease. CW1 yielded two digestion products of molecular weights $22 \times 10^{3}$ and $18 \times 10^{3}$ and $\mathrm{CW} 2$ one product of molecular weight $14 \times 10^{3}$. Products of the same molecular weights were found for the co-migrating extracellular polypeptides.

\section{DISCUSSION}

Although certain alleles and various treatments may induce the formation of haploid fruit-bodies of Schizophyllum commune (Leslie \& Leonard, 1979; Esser et al., 1979), the establishment of the dikaryon is a trigger for rapid and normal fruit-body initiation and development (Schwalb, 1978). The strains of S. commune (co-isogenic monokaryons 4-39 and 440) and the derived dikaryon used in the present investigation provide exceptionally good examples of such behaviour because upon plating mycelial macerates the dikaryon is completely covered with primordia within $100 \mathrm{~h}$ while no primordia are formed in the monokaryons. The cup-shaped primordia do not develop large pilei but their synchronous and abundant appearance allows for an analysis of associated molecular changes. In addition, any difference found between the monokaryons and the dikaryon under conditions where the latter mycelial type forms fruit-bodies, can be regarded as ultimately based on the interactions of the incompatibility genes that governed the establishment of the dikaryon.

First of all, the incompatibility factors determine the transition from the monokaryotic to the dikaryotic state. The molecular events associated with this transition can best be studied by comparing co-isogenic monokaryons and the derived dikaryon when grown in shaken cultures. The co-isogenic monokaryons differ only in the alleles of their incompatibility factors while the effects of combining the different alleles should be shown in the dikaryon with its distinct hyphal morphology.

In an analysis using pulse-labelled proteins and two-dimensional electrophoresis De Vries et al. $(1980 a)$ found that the two monokaryons produced identical protein patterns but that in the dikaryon there was an apparent shift in the position of some 20 polypeptides among 700 polypeptides located on the gels. This is a small difference compared to previous claims of extensive differences in accumulated proteins and isozymes but a reappraisal by Evers $\&$ Ross (1983) of these early experiments has shown that the findings must have been in error. 
In contrast to our previous analysis (De Vries et al., 1980a) we now used hot-SDS extraction instead of urea-Nonidet extraction at $25^{\circ} \mathrm{C}$ and fluorography to detect labelled proteins in the gels. Among $\mathbf{4 0 0}$ most intensely labelled proteins, the differences between monokaryons and dikaryon grown in shaking culture were even smaller (data not shown) than those previously reported, but the $4 \mathrm{~d}$ old surface cultures showed distinct differences. In the dikaryon 37 polypeptides not seen in the monokaryons were present, while only eight polypeptides appeared specific for the monokaryons.

These differences in the patterns are strikingly in accordance with predictions made on the basis of the analysis of mRNA divergence (Hoge et al., 1982b). This holds both for the number of additional polypeptides synthesized in the fruiting dikaryon (37) as for the absence of a substantial number of polypeptides specifically synthesized in the monokaryons. However, due to post-transitional modification of the polypeptides (De Vries et al., 1980 b) it was impossible to equate the individual in vivo polypeptides with the translation products by their position on the gels.

Most of the additional polypeptides in the dikaryon were already being synthesized at day 3 , marked by the formation of hyphal aggregates but before differentiation of the cup-shaped primordia that occurred on day 4 . At that time 10 of the additional polypeptides were being synthesized exclusively in the primordia.

Among the polypeptides specifically synthesized by the fruiting dikaryon, a relatively large number (nine) were excreted into the medium. In fact, the most pronounced differences in patterns of protein synthesis between monokaryons and fruiting dikaryon were found when extracellularly excreted polypeptides were analysed. Significantly, these patterns were very similar when the two mycelial types were compared while growing in liquid shaken culture, so that at least a correlation exists between the additional polypeptides excreted by the dikaryon and the process of primordia formation as occurring in surface cultures. The nature of the extracellular polypeptides specifically excreted by the fruiting dikaryon is open to speculation, but among them may be polypeptides which constitute the phenol oxidases that have been implicated in the initiation of reproductive structures in a variety of fungi (cf. Leatham \& Stahmann, 1981) including fruit-bodies in S. commune (Leonard \& Phillips, 1973). On the basis of their apparent molecular weight, iso-electric point, and partial digestion fragments, two of the extracellular polypeptides (CW1 and CW2) were probably identical to polypeptides also firmly associated with hyphal walls prepared from the fruiting dikaryon.

The finding that hyphal walls prepared from fruiting dikaryotic cultures contain two extra polypeptides (CW1 and CW2) as well as at least six shared with hyphal walls from monokaryotic cultures is of considerable interest in view of the possibility that these two polypeptides are involved in the binding of adjacent hyphae in the developing fruit-bodies. Significantly, when the fungi were grown vegetatively in shaken liquid culture, polypeptides CW1 and CW2 were absent from the walls of the dikaryon, which then only contained the six polypeptides common to both forms. Characteristic for vegetative growth of a fungal mycelium while colonizing the substrate is an avoidance reaction of the growing hyphae (Bull \& Trinci, 1977). In fruit-body primordia however, there is an apparent binding of adjacent hyphae together, suggesting a change at the hyphal wall surface. Such a change has been indicated by specific staining of the wall surface in aggregating hyphae in $S$. commune (Wessels, 1965) and by electron microscopical observations (Van der Valk \& Marchant, 1978) in both S. commune and Coprinus cinereus. Specific polypeptides as detected in the walls of the aggregating dikaryon may be involved directly in the adhesion of hyphae, as in agglutination in Hansenula wingii (Crandall et al., 1977) or indirectly as suggested for phenol oxidases in oxidative cross linking of wall components of adjacent hyphae (Bu'lock, 1967; Leatham \& Stahmann, 1981). The fact that these polypeptides accumulate in the walls and apparently are also excreted into the culture medium suggests that they could be isolated in sufficient quantities to allow for a functional analysis.

We acknowledge the assistance of Geert Liebe, Hilda Folkerts and Jan Aten in doing a number of the experiments and Dr P. E. Long (University of Leicester, UK) for stimulating discussions on differences in cellwall proteins involved in aggregation of hyphae. 


\section{REFERENCES}

Bull, A. T. \& TrincI, A. P. J. (1977). The physiology and metabolic control of fungal growth. Advances in Microbial Physiology 50, 1-84.

Bu'Lock, J. D. (1967). Fungal metabolites with structural function. In Essays in Biosynthesis and Microbial Development, E. R. Squibb Lectures on Chemistry of Microbial Products, pp. 1-18, New York: Wiley.

Cleveland, D. W., Fischer, S. G., Kirschner, M. W \& Laemmli, U. K. (1977). Peptide mapping by limited proteolysis in sodium dodecyl sulfate and analysis by gel electophoresis. Journal of Biological Chemistry 252, 1102-1106.

Crandall, M., Egel, R. \& Mackay, V. L. (1977). Physiology of mating in three yeasts. Adiances in Microbial Physiology 15, 307-398.

De VRIEs, O. M. H., Hoge, J. H. C. \& Wessels, J. G. H. $(1980 a)$. Regulation of the pattern of protein synthesis in Schizophyllum commune. Developmental Biology 74, 22-36.

De Vries, O. M. H., Hoge, J. H. C. \& Wessels, J. G. H. (1980b). Translation of RNA from Schizophyllum commune in a wheat germ and rabbit reticulocyte cell-free system. Comparison of in vitro and in vivo products after two-dimensional gel electrophoresis. Biochimica et biophysica acta 607, 373-378.

Dons, J. J. M., De VRIES, O. M. H. \& Wessels, J. G. H. (1979). Characterization of the genome of the basidiomycete Schizophyllum commune. Biochimica et biophysica acta 563, 100-112.

Esser, K., Saleh, F. \& Meinhardt, F. (1979). Genetics of fruit-body production in higher basidiomycetes. II. Monokaryotic and dikaryotic fruiting in Schizophyllum commune. Current Genetics 1, 85-88.

EVERS, D. C. \& Ross, I. K. (1983). Isozyme patterns and morphogenesis in higher basidiomycetes. Experimental Mycology 7, 9-16.

Hoge, J. H. C., Springer, J., Zantinge, B. \& WESSELS, J. G. H. (1982a). Absence of differences in polysomal RNAs from vegetative monokaryotic and dikaryotic cells of the fungus Schizophyllum commune. Experimental Mycology 6, 225-232.

Hoge, J. H. C., Springer, J. \& Wessels, J. G. H $(1982 b)$. Changes in complex RNA during fruitbody initiation in the fungus Schizophyllum commune. Experimental Mycology 6, 233-243.

LasKey, R. A. \& Mills, A. D. (1975). Quantitative film detection of ${ }^{3} \mathrm{H}$ and ${ }^{1+} \mathrm{C}$ in polyacrylamide gels by fluorography. European Journal of Biochemistr. 56, 335-341.
Leatham, G. F. \& StahmanN, M. A. (1981). Studies on the laccase of Lentinus edodes: specificity, localization and association with the development of fruiting-bodies. Journal of General Microbiology 125, 147-157.

Leonard, T. J. \& Phillips, L. E. (1973). Study of phenoloxidase activity during the reproductive cycle in Schizophyllum commune. Journal of Bacteriology 114, 7-10.

Leslie, J. F. \& Leonard, T. J. (1979). Three independent genetic systems that control initiation of a fungal fruit-body. Molecular and General Genetics 171, 257-260.

O'FARRELl, P. H. (1975). High resolution two-dimensional electophoresis of proteins. Journal of Biological Chemistry 10, 4007-4021.

Pringle, J. R. (1975). Methods for avoiding proteolytic artefacts in studies of enzymes and other proteins from yeasts. Methods in Cell Biology 12 , 149-184.

Schwalb, M. N. (1978). Regulation of fruiting. In Genetics and Morphogenesis in the Basidiomycetes, $\mathrm{pp}$ 135-165. Edited by M. N. Schwalb \& P. G. Miles New York: Academic Press.

VAN DER VALK, P. \& MARChANT, R. (1978). Hyphal ultrastructure in fruit-body primordia of the basidiomycetes Schizophyllum commune and Coprinus cinereus. Protoplasma 95, 57-72.

Volz, P. A. \& Niederpruem, D. J. (1970). Induced cytological aberrancies in basidiocarp morphology of Schizophyllum commune. Archives of Microbiology 72, 371-374.

Wessels, J. G. H. (1965). Morphogenesis and biochemical processes in Schizophyllum commune $\mathrm{Fr}$. Wentia 13, 1-113.

Wray, W., Boulikas, T., Wray, V. P.\& Hancock, R. (1981). Silver staining of proteins in polyacrylamide gels. Analytical Biochemistry 118, 197-203.

Zantinge, B., Dons, H. \& Wessels, J. G. H. (1979). Comparison of poly (A)-containing RNAs in different cell types of the lower eukaryote Schizophyllum commune. European Journal of Biochemistry 101, 251 260.

Zantinge, B., Hoge, J. H. C. \& Wessels, J. G. H. (1981). Frequency and diversity of RNA sequences in different cell types of the fungus Schizophyllum commune. European Journal of Biochemistry 113, 381 389 